\title{
Occurrence and characterisation of naphthenic acid fraction components in oil residues stranded on the shoreline of Chedabucto Bay, Nova Scotia, Canada
}

\section{Zeyu Yang ( $\sim$ yangzeyu72@163.com )}

Emergencies Science and Technology Section https://orcid.org/0000-0002-2329-9610

\section{Gong Zhang}

Environment and Climate Change Canada

Chun Yang

Environment and Climate Change Canada

Keval Shah

Environment and Climate Change Canada

\section{Bruce Hollebone}

Environment and Climate Change Canada

\section{Sonia Laforest}

Environment and Climate Change Canada

\section{Patrick Lambert}

Environment and Climate Change Canada

\section{Carl Brown}

Environment and Climate Change Canada

\section{Research Article}

Keywords: naphthenic acid fractions, Chedabucto Bay, occurrence, fate and behaviour

Posted Date: August 5th, 2021

DOI: https://doi.org/10.21203/rs.3.rs-300363/v1

License: (c) (1) This work is licensed under a Creative Commons Attribution 4.0 International License. Read Full License 


\section{Abstract \\ Purpose}

This study aimed to investigate the occurrence, fate, and behaviour of oil components 46 years post-spill by analysing polar naphthenic acid fraction components (NAFCs).

\section{Methods}

Oil residues and sediment samples were collected from the shoreline of Chedabucto Bay, Nova Scotia, Canada, at sites affected by the 1970 Arrow oil spill, in June 2016. Sediments were extracted with Soxhlet extraction; NAFCs were separated through a silica gel column firstly, then analysed by high-performance liquid chromatography-high-resolution mass spectrometry (HPLC-HRMS).

\section{Results and discussion}

In all samples, the most abundant NAFC species were those having only oxygen as the heteroatom $\left(O_{0}\right.$ species, o indicating the number of oxygen atoms, from 1 to 8 ) and a high degree of saturation. $\mathrm{O}_{2}$ species accounted for $50-70 \%$ of all $O_{0}$ species. NAFCs with sulphur heteroatoms were the second abundant species, then by those containing both nitrogen and oxygen heteroatoms. NAFCs in most Arrow oil residues had higher levels of oxygen species, especially for heavier molecular weight congeners, than the oil remaining stored in the sunken tanker. Environmental sediment samples, collected from near a marina away from the spill site, mainly contained a biological contribution with obviously recent petroleum oil input, whereas all Arrow oil residues were composed mainly of NAFCs that are characteristic of petroleum, and varied abundance between sites.

\section{Conclusions}

Characteristics of NAFCs from different sources varied from each other. Site-specific environmental conditions played a major role in controlling the fate and behaviour of oil components, including NAFCs.

\section{Introduction}

Chedabucto Bay, Nova Scotia, Canada, is an important maritime transportation area with ports that serve as hubs for surrounding industries and regional transportation networks. In 1970, the tanker Arrow sank on Cerberus Rock $\left(45.47^{\circ} \mathrm{N}, 61.10^{\circ} \mathrm{W}\right)$. Of the total oil spilled from the wreck, approximately $2000 \mathrm{~m}^{3}$ of Bunker $\mathrm{C}$ fuel was deposited along the southern shores of Chedabucto Bay. Ninety percent of the spilled oil was not recovered, and much of this fraction reached the beaches and coastlines in Guysborough, Richmond, and Cape Breton counties, including sites as far east as Glace Bay, Lingan, and Dominion 
(Vandermeulen \& Buckley 1985). Oil residues were deposited on the shore surface or entered into the sediments after the incident (Owens et al. 1994, Vandermeulen \& Singh 1994).

In the years following the Arrow sinking, a series of studies have investigated the fate, behaviour, and environmental effects of the spilled oil (Owens et al. 1994, Owens et al. 2008b). These studies reported that oil penetrated deep into the sediment to remain as preserved layers or formed asphalt pavement, which eventually became buried under sediment (Owens 1978). The natural attenuation of oil residues varied depending on location (Thomas 1977). At sites where the oil was deeply buried within the sediment-a very low energy environment-residual oil persisted over 20 years in the environment from 1970 to 1990 (Vandermeulen \& Gordon 1976, Vandermeulen \& Singh 1994). Some subsurface oil deposits, however, became re-exposed because of storms or strong wave action (Owens et al. 2008a, Owens et al. 2008b).

As part of the long-term monitoring of the spilled Arrow oil, samples have been collected 22, 23-30, and 35 years post-spill (Lee et al. 2003, Owens et al. 2008a, Wang et al. 1994). Substantial biodegradation has depleted most resolved components, such as $n$-alkanes and polycyclic aromatic hydrocarbons (PAHs), particularly within the near-surface deposits. Some triterpenes, hopanes, and steranes persisted as relatively conservative biomarkers. Subsurface residues remain sequestered and unaltered. The degradation of these oil residues required substantial changes to the environmental conditions along the local shoreline.

In the summer and fall 2015, oil leaked from the sunken vessel. This released oil re-covered some sections of local shoreline. In June 2016, Environment and Climate Change Canada (ECCC) launched a shoreline survey of the shoreline of Chedabucto Bay. The survey aimed to investigate the status of the recent oil contamination and the degree of weathering (46 years post-spill) of oil residues from the 1970 Arrow spill. These investigation relied on the analysis of total petroleum hydrocarbons (TPH), $n$-alkanes, petroleum-related biomarkers, polycyclic aromatic hydrocarbons (PAHs), and their alkylated congeners (APAHs)(Yang et al. 2018). Various weathering processes had contributed to the ageing of these oil residues with microbial degradation being the dominant process. The extent of weathering and alteration of oil residues varied depending on environmental conditions; therefore, evaluating the actual environmental exposure time from specific oil residues remained difficult.

The earlier studies of oil residues from the Arrow (Lee et al. 2003, Owens et al. 2008a, Owens et al. 2008b, Vandermeulen \& Singh 1994, Wang et al. 1994) investigated only components detectable by gas chromatography (GC). In addition to these GC-detected targets, there exist many oxygenated intermediates, which are produced through the biodegradation and (or) photo-oxidation of oil. These intermediates are usually characterised by low volatility and high polarity because of the addition of oxygen. Naphthenic acid fraction compounds (NAFCs), a class of these oxygenated intermediates, contain a diverse family of carboxylic acids and other acid-extractable organic compounds that vary in structure (e.g., aromatic, adamantane, or diamondoid structures) and species (e.g., sulphur- and nitrogencontaining compounds and multiple oxygenated acids)(Rowland et al. 2011). The marked water 
solubility and, therefore, high bioavailability of NAFCs heighten the relative toxicity of these compounds when compared with most non-polar oil hydrocarbons. The toxicity of NAFCs has been noted, in particular, in the processed waters of oil sands (Clemente \& Fedorak 2005, Frank et al. 2009, Frank et al. 2008, Vanderveen et al. 2017).

The degradation of oil components increases the abundance of NAFCs within oil-water mixtures (Ray et al. 2014, Yang et al. 2017b). Studying the variability of these polar-oxygenated components of petroleum mixtures provides an alternative means of fully understanding the fate and behaviour of oil components in the environment. It can also help us identify the weathering mechanisms of oil after a spill.

In this study, samples collected from Chedabucto Bay, Nova Scotia, were analysed by high-performance liquid chromatography-ultra-high-resolution Orbitrap mass spectrometry combined with electrospray ionisation in the negative mode (HPLC-HRMS) to identify NAFCs. We investigated the variability of the chemical composition of NAFCs among sample sites, and provided the compositional characteristics of polar NAFCs. These analyses provided a better understanding of the fate of spilled oil in relation to natural attenuation and served as a tool for identifying petroleum sources. No certified reference materials exist, as of yet, for identifying and quantifying NAFCs because of the complex chemical composition of these compounds. We therefore generated average mass spectra to identify NAFCs on the basis of their elemental composition within each sample. All values reported in this study must therefore be regarded as semi-quantitative because the reported results are the relative abundance normalised to the response of an internal standard and the weight of oil/total solvent extractable materials (TSEM) in each sample.

\section{Experimental Section}

\subsection{Sampling}

The sampling strategies and sample information are described in Yang et al.(Yang et al. 2018) In this study, we analysed nine environmental samples (collected from sites located between $45.268^{\circ} \mathrm{N}$, $61.022^{\circ} \mathrm{W}$ and $45.615^{\circ} \mathrm{N}, 61.365^{\circ} \mathrm{W}$ ) as well as two additional oil samples. Most of the nine environmental samples were oil mixed with sediment or were oil residues recovered from the surface of rocks or from under or between rocks. They included two oil residues from Cape Auguet, four oil residues from Black Duck Cove, one oil residue from Indian Cove, and two sediments from the marina at Port Hawkesbury (see Table 1 for more details of the samples). An initial investigation demonstrated that recently released petroleum had contaminated both samples recovered from the Port Hawkesbury area(Yang et al. 2018); the seven remaining samples represented historical (1970) contamination from the sunken Arrow. For this study, two additional oil samples were collected and analysed for comparative purposes: (i) an Arrow oil sample recovered directly from the sunken tanker, and (ii) a naturally weathered oil residue sample collected from a site that was re-oiled by the release of Arrow oil in 2015. The Canadian Coast Guard collected and provided both of these samples.

\section{$2.2 \quad$ Chemicals and materials}


Most chemicals and standards were supplied by Sigma-Aldrich (Bellefonte, PA, USA) and Sigma-Aldrich (Oakville, ON, Canada), including LC/MS-purity acetic acid, formic acid, ammonium acetate, HPLC grade methanol, isopropanol, acetonitrile, and deuterated fatty acids (such as $\left[{ }^{2} H_{15}\right]$ caprylic acid (C8:0- $\left.d_{15}\right)$, $\left[{ }^{2} \mathrm{H}_{2}\right]$ palmitic acid- (C16:0- $\left.d_{2}\right)$, and $\left[{ }^{2} H_{27}\right]$ myristic acid (C14:0- $\left.d_{27}\right)$ ). All other solvents used are of the highest purity (Caledon, Georgetown, ON, Canada). Ultra-pure water was prepared from a Milli-Q water purification system (Millipore, Billerica, MA, USA). Silica gel (100-200 mesh) was supplied by Spectrum Chemicals (Gardena, CA, USA).

\subsection{Sample extraction, clean-up, and analysis}

Sample extraction procedures followed the methods presented in a previous study(Yang et al. 2017a). In brief, an aliquot of sediment (approximately $10 \mathrm{~g}$ wet weight), thoroughly mixed with sodium sulphate, was spiked with an appropriate amount of deuterated fatty acids as surrogates, including octanoic acid $\left(\mathrm{C} 8: 0-d_{15}\right)$ and eicosanoic acid $\left(\mathrm{C} 20: 0-d_{39}\right)$. The mixture was then Soxhlet-extracted with DCM for $16 \mathrm{~h}$. Oil samples were diluted directly with DCM. Appropriate amounts of diluents, spiked with the same surrogates as the sediment, were then transferred for fractionation and (or) clean-up. The oil residue samples were ultra sonicated consecutively three times with DCM. Appropriate extracts or diluents were loaded into a column ( $5 \mathrm{~mm}$ i.d.) with $1 \mathrm{~g}$ of silica gel topped with $1 \mathrm{~cm}$ of anhydrous $\mathrm{Na}_{2} \mathrm{SO}_{4}$. Ten millilitres of DCM was used to remove interference from the target components. Another $7 \mathrm{~mL}$ of methanol containing $0.1 \%$ formic acid was used to elute the polar targets. The eluted material was then dried by gentle $N_{2}$ and reconstituted in $1 \mathrm{~mL}$ of HPLC grade isopropanol, using $1.0 \mu \mathrm{g} / \mathrm{mL}$ of deuterated palmitic acid $\left(d_{31}-\mathrm{C} 16: 0\right)$ as an internal standard. All samples were centrifuged at $3000 \mathrm{rpm}$ for $10 \mathrm{~min}$ prior to HPLC-HRMS analysis.

\section{$2.4 \quad$ HPLC-HRMS analysis of NAFCs}

The analytical methods were adapted from Zhang et al(Zhang et al. 2014). Briefly, sample analysis was performed using an Accela HPLC system paired with an Exactive Orbitrap Mass System (Thermo Fisher Scientific, San Jose, CA, USA) with the electrospray ionisation (ESI) source in a negative-ion mode. A 20 min LC gradient method was used for separation with a Poroshell $120 \mathrm{EC}-C_{8}$ column $(100 \times 2.1 \mathrm{mmi} . \mathrm{d}$., $2.7 \mu \mathrm{m}$, Agilent) at a flow rate of $0.25 \mathrm{~mL} \cdot \mathrm{min}^{-1}$. The gradient method was programmed among three mobile phases: mobile phase $A$, water with $0.0025 \%$ of formic acid; mobile phase $B, 95 \% / 5 \%$ acetonitrile/water with $3 \mathrm{mmol} / \mathrm{L}$ ammonium acetate; and mobile phase $\mathrm{C}, 85 \% / 10 \% / 5 \%$ isopropanol/toluene/water with $3 \mathrm{mmol} / \mathrm{L}$ ammonium acetate. Initially, the $95 \%$ mobile phase A and $5 \%$ mobile phase $B$ were held isocratically for $2 \mathrm{~min}$, followed by a linear increase from $5 \%$ to $100 \%$ of the mobile phase B over 8 min. Over the following 5 min, the mobile phase B decreased to $10 \%$; the mobile phase $C$ increased linearly to $90 \%$. Both mobile phases were held at these percentages for $10 \mathrm{~min}$. Mobile phase $B$ increased to $100 \%$ over 30 seconds, and was then held at this percentage for 90 seconds. After this, the mobile phase B decreased to $5 \%$. Mobile phase A increased to $95 \%$ over 30 seconds and was held at this level for 5 min prior to the next injection. The Orbitrap mass spectrometer was operated in ESI 
negative mode (ESI-). Data were acquired in full scan with a mass to charge $(\mathrm{m} / \mathrm{z})$ ratio ranging from 80 to 1600 . The negative ion of the acetic acid dimer $(2 M-1, m / z=119.03498)$ was used as a lock mass for scan-to-scan calibration correction to ensure $<2 \mathrm{ppm}$ mass accuracy. The mass parameters were set at a spray voltage of $4.0 \mathrm{kV}$ and a capillary temperature of $320^{\circ} \mathrm{C}$; the settings for the tube lens, sheath gas and auxiliary gas were set at $-90 \mathrm{~V}, 45 \mathrm{~mL} \cdot \mathrm{min}^{-1}$, and $15 \mathrm{~mL} \cdot \mathrm{min}^{-1}$, respectively.

Heteroatom-containing species, including those having a formula of $C_{\mathrm{c}} H_{\mathrm{h}} N_{\mathrm{n}} \mathrm{O}_{\mathrm{o}} S_{\mathrm{s}}$, where c, h, n, o, and $s$ are the elemental numbers in the formula $(6 \leq \mathrm{c} \leq 160,6 \leq \mathrm{h} \leq 122,0 \leq 0 \leq 8,0 \leq \mathrm{n}$, and $\mathrm{s} \leq$ 2), were detected as deprotonated molecules, which are $[\mathrm{M}-\mathrm{H}]^{-}$ions, by the Orbitrap MS ESI-scan. Spectral interpretation of the NAFCs was obtained to retrieve the average mass spectra over a retention time of 5 to 40 minutes. Elemental formulae were generated at a mass accuracy of $2 \mathrm{ppm}$ and assigned on the basis of $\mathrm{m} / \mathrm{z}$ values by the Xcalibur software package (Thermo Fisher Scientific, San Jose, CA, USA). The MS data were processed; the elemental composition of the compounds was determined by measuring the accurate $\mathrm{m} / \mathrm{z}$ values. A resolving power $\left(\mathrm{m} / \mathrm{Dm}_{50 \%}\right)$ of approximately 100,000 and a mass accuracy of $<2$ ppm provided unambiguous molecular formula assignments for the single-charged molecular ions. Double-bond equivalent (DBE) values represent the number of rings and the number of carbon double bonds in a given molecular formula, which is calculated using equation (Eq. 1) for the elemental formula of $\mathrm{C}_{\mathrm{c}} \mathrm{H}_{\mathrm{h}} \mathrm{N}_{\mathrm{n}} \mathrm{O}_{\mathrm{o}} S_{\mathrm{s}}$ (McLafferty \& Turecek 1993):

$$
D B E=c-\frac{h}{2}+\frac{n}{2}+1
$$

Isotopes, adducts, and minors were excluded from NAFC analysis. Because there are no weatheringresistant biomarkers for the Orbitrap MS available for normalising the data set, the responses for $\mathrm{m} / \mathrm{z}$ peaks, relying on the assigned molecular formulae, were normalised to the abundance of the internal standard and oil/TSEM weight of each mass spectrum. This normalisation can partially eliminate detection bias in the ESI response, caused by sample matrix effects and/or ionisation suppression effects. Ionisation suppression will produce an apparent reduction in analyte recovery from different matrices when the ESI is used in a negative ion mode(Page et al. 2007).

\subsection{Quality control and quality assurance}

Sodium sulphate, spiked with an equal amount of surrogates, was prepared for the blank control test. The same analytical procedures were run through the blank controls to evaluate the background contribution to NAFC analysis. All reported NAFC data were background corrected. The surrogate recoveries were determined as $83 \pm 18 \%$ for $\mathrm{C} 8: 0-d_{15}$ and $87 \pm 20 \%$ for $\mathrm{C} 20: 0-d_{39}$, when compared with the response of the authentic standards.

\section{Results And Discussion}




\subsection{Chemical characteristics of acidic extractable components}

All total ion chromatograms (TICs) in negative-ion ESI mode for the representative samples are presented in Fig. S1. The TICs represent the total acidic components present in the samples, which may contain sulphur, oxygen, and/or nitrogen heteroatoms. Visualisation analysis indicated that the abundance of acidic extracts varied between samples. For example, the NAFC composition in the two source oils differed somewhat. A greater abundance of resolved and unresolved peaks was eluted prior to 23 min for Sample 3281, which was collected directly from the sunken tanker in 2015 , relative to Sample 3282 , the weathered oil residue from the 2015 spill. Sample 3281 also produced more unresolved peaks between 23 and 29 min than Sample 3282.

The $C_{8}$ column was used for liquid chromatographic separation in this study. Hydrophobic molecules in the polar mobile phase tend to adsorb to the hydrophobic $C_{8}$ stationary phase, whereas hydrophilic molecules in this phase pass through the column faster than the hydrophobic molecules. The relatively higher abundance of the earlier eluted peaks attests to acidic components, which have a higher polarity, being more abundant in Sample 3281 than in Sample 3282. This pattern is expected as Sample 3282 was weathered after being spilled into the environment, and Sample 3281 remained stored in the sunken vessel.

The pair of Arrow oil residues collected from Cape Auguet (Fig. S1b) and the four Black Duck Cove samples (Fig. S1C) presented similar patterns at the various retention times. Specifically, a hump having clearly resolved peaks in the TIC was present prior to $23 \mathrm{~min}$, and another hump, lacking obvious resolved peaks, occurred between 23 and $31 \mathrm{~min}$. The abundance varied between the samples in some cases. Two oil residues from Black Duck Cove (Samples 3184 and 3186) differed from each other despite both being sampled from the upper tidal zone at different sites. Sample 3184 was collected from rock surfaces, whereas Sample 3186 was obtained from under rocks. As shown by Yang et al. (Yang et al. 2018), certain high molecular weight $n$-alkanes were present in Sample 3184, but they were not observed in Sample 3186. Both samples from Cape Auguet were collected from under rocks; the TIC spectra of both illustrated a very similar abundance upon visual inspection. The two samples from the Port Hawkesbury marina area also presented similar NAFC patterns for the various retention times. The spectra of both Port Hawkesbury samples had more resolved peaks and higher abundances than the above-mentioned oil and oil residues. This was especially true for Sample 3247. For the Port Hawkesbury samples, lighter molecular weight NAFCs were dominant. Nonetheless, abundances differed between the two samples, a dissimilarity that could be ascribed to discrepancy in either sample weight and/or the specific sampling site.

Figure 2 presents the average spectra for several representative samples. NAFC abundance and peak numbers in all Arrow oil residues (except Sample 3282) were higher than those of Sample 3281, the stored source oil. Sample 3282 presented a limited number of peaks, in particular when the $\mathrm{m} / \mathrm{z}$ was < 
400. Most samples had a lower abundance for components at $\mathrm{m} / \mathrm{z}>400$ than the Arrow source oil. Visual inspection of the average spectra revealed that the most abundant peaks did not shift to a lower/higher mass for the heavily weathered or even partially weathered Arrow oil. This lack of change prevents the identification of the source Arrow oil from the oil residues.

Similarly, the two samples from the marina at Port Hawkesbury area (Fig. 2d) had fewer peaks compared with most Arrow oil residues and the source oil. This was particularly true toward the light end, which showed marked differences from most Arrow oil residues. This discrepancy occurred as the sources of these identified acidic components were not identical. As concluded by Yang et al.(Yang et al. 2018), the identified $n$-alkanes of the pair of samples from Port Hawkesbury reflected terrestrial input as the major source with a minor contribution from petroleum hydrocarbons. APAH analysis also showed naphthalenes and phenanthrenes to be the most abundant families; therefore, oil contamination in these two samples was lighter and fresher than all oil residues from the Arrow. The Port Hawkesbury samples, therefore, have a lower abundance of NAFCs, as these samples were contaminated by more recently released petroleum(Yang et al. 2017b). Similar to the weathered tars, oxygenated intermediates, like acidic components and in particular heavy molecular weight components, should increase with weathering(Katarina Huba \& Gardinali 2016). On the other hand, NAFCs are oxygenated intermediates. Further degradation can markedly decrease their abundance in strongly weathered oil. Identified NAFCs in some samples can also reflect biogenic sources. This is particularly true for Samples 3282, 3247, and 3251 , each presenting several abundant peaks when $\mathrm{m} / \mathrm{z}<300$.

Because of possible background interference, variable sample weights, and/or ionisation efficiencies of ESI between samples, we discuss, in the following section, the specific acidic classes that contain various heteroatoms after normalising them by the abundance of the internal standard and the oil or TSEM weight.

\subsection{Distribution of total heteroatom classes in the samples}

HRMS can identify various petroleum components on the basis of exact masses, and generate the corresponding molecular formula. Thus, identification of NAFCs in our study relies mainly on the chemical composition provided by HRMS. This situation arises because of the poor separation efficiency of NAFC congeners/isomers by LC and the limited commercial availability of authentic NAFC standards. We grouped compounds into classes within a sample on the basis of their heteroatom content by using the assigned molecular formula for each $\mathrm{m} / \mathrm{z}$ peak. The most abundant acidic heteroatom class for all samples represented components containing only the oxygen heteroatom. Herein, these species-species with the formula $C_{\mathrm{c}} H_{\mathrm{h}} \mathrm{O}_{\mathrm{o}}$-are abbreviated as $O_{\mathrm{o}}$ species. $O_{\mathrm{o}}$ species accounted for $57-76 \%$ of the total acidic components among all samples, followed by $N_{\mathrm{n}} S_{\mathrm{s}} \mathrm{O}_{\mathrm{o}}$ species, which accounted for $18-33 \%$ of the total acidic components. Other species provided a minor contribution to total NAFC (Fig. S2).

The abundance and distribution of heteroatom classes varied between samples. In terms of heteroatom abundance of the various samples-Fig. 3 presents major species having a $>0.1 \%$ abundance of the internal standard in each injection in the ESI (-)-Orbitrap MS. he two source oils had a lower abundance 
than other samples; this was particularly true for the weathered oil sample spilled in 2015 (Sample 3282, Fig. 3a). Sample 3247, collected from the marina in Port Hawkesbury area, had the highest abundance of $O_{0}$ species (Fig. $3 \mathrm{~d}$ ). The abundance of $O_{\mathrm{o}}$ species then decreased in order of the two samples from Cape Auguet (Fig. 3b), a sample from Indian Cove (Fig. 3b), and a sample from Black Duck Cove (Fig. 3c). The

samples from Port Hawkesbury (Fig. 3d) and Indian Cove (Sample 3216 in Fig. 3b) contained the highest abundance of $N_{n} S_{s} O_{0}$ species, followed by Sample 3179 from Black Duck Cove (Fig. 3c), then the remaining oil residues. Given that the abundance of these semi-quantitative species varied between sampling sites within the same area, we can infer that sampling site plays an important role in controlling the fate of these identified NAFCs, a finding similar to that from the analysis of conventional petroleum hydrocarbons(Yang et al. 2018).

The elemental composition of these acidic species varies in terms of carbon, sulphur, oxygen, and nitrogen numbers, as well as the number of double bonds/naphthenic rings. Each ring or double bond in a molecule reduces the number of hydrogen atoms by two (Lemkau et al. 2014). DBE, representing the number of rings plus double bonds, is a useful parameter for examining the compositional differences between samples. As $O_{\mathrm{o}}$ species formed the most abundant group in all major classes, in the following section, we address the distribution profiles of total $O_{0}$ species versus carbon number, DBE values, and oxygen numbers between samples.

\subsection{Characteristics of $O_{0}$ species}

\subsection{1 $\mathrm{O}_{\mathrm{o}}$ species versus carbon number}

In plots of the relative abundance of subtotal $O_{0}$ species versus carbon number $\left(C_{6}\right.$ to $\left.C_{60}\right)$, the Arrow source oil and most Arrow oil residues presented a typical single bell shape, having a maximum of species at carbon numbers 21 to 24 (Fig. 4). Several samples (Samples 3282, 3179, 3247, and 3251), however, presented double peaks. More specifically, the most abundant peak occurred at carbon numbers 16 to 22 with a strong predominance of even-over-odd carbon numbers. The second abundant peak, occurring around carbon number 30 , did not show an obvious predominance of even-over-odd carbon numbers. The importance of short-chain compounds and the strong predominance of even-over-odd carbon numbers at carbon numbers 16 to 22 indicate that biological inputs represent a major source in these samples (Cheung et al. 2010, Dunn et al. 2008). At the same time, the presence of abundant longchain compounds lacking a clear predominance of even-over-odd carbon numbers indicates that another source is responsible for the petroleum input(Headley et al. 2016).

As discussed above, $O_{\mathrm{O}}$ species abundance with carbon number varied between samples, regardless of the existing patterns. For single bell-shaped samples, the carbon numbers of the most abundant $O_{\mathrm{o}}$ species differed between the source oil and the weathered Arrow oil residues. For example, Sample 3281, the source oil, had a maximum abundance at carbon number 21, whereas all other oil residues produced a maximum abundance peak at carbon number 24 . 
Lower molecular weight NAFCs are considered to be more biodegradable and more water soluble than higher molecular weight NAFCs(King et al. 2014, Ray et al. 2014). These weathering recalcitrant NAFCs will accumulate in oil residues through long-term natural weathering; therefore, oil residues that have experienced depletive weathering processes should have heavier molecular weight $O_{0}$ species than the species of the original source oil. Samples having a double peak shape of the $O_{0}$ species, e.g., Samples 3282 and 3179, reflected a mixed source of biological contributions and Arrow oil residues. In these two latter samples, long-chain $O_{\mathrm{o}}$ species were more abundant than those in Samples 3247 and 3251 . This pattern reflects a greater amount of $O_{0}$ species being sourced from the Arrow oil residues, e.g., in Samples 3282 and 3179. The petroleum contamination in Samples 3247 and 3251 was sourced mainly from recent human activities rather than the original Arrow spill(Yang et al. 2018). In these two samples, the predominance of the light $O_{\mathrm{o}}$ species with an obvious even-to-odd preference suggests a biological contribution, whereas those $O_{0}$ species having a longer carbon-chain length represent more recent oil contamination.

The plots of the percentages of $O_{\mathrm{o}}$ species against carbon numbers, normalised to total $O_{0}$ species in different samples, are shown in Fig. S3. For the spectra having a single bell shape, $O_{\mathrm{O}}$ species attained a maximum of $5-6 \%$ at carbon numbers 21 and 24 . Maximum peaks in samples reflecting a biological contribution (i.e., Samples $3282,3179,3247$, and 3251 ) accounted for $8-13 \%$ of total $O_{0}$ species for carbon numbers 16 and 20 . The second peak accounted for 2-5\% around carbon number 30 .

\subsection{2 $O_{o}$ species versus DBE}

For all eleven samples, two patterns emerged in the plots of the relative abundance of total $O_{0}$ species versus DBE (Fig. 5). The first pattern had $O_{0}$ species with a DBE of 2-4 (1-3 cyclic rings) as the most abundant group in the source oil, Sample 3281, and most environmental oil residues (e.g., Samples 3216, 3163,3165 , and all oil residues from Black Duck Cove). These congeners accounted for $20-28 \%$ of total NAFCs (Fig. S4). Then their abundance rapidly decreased for DBE numbers 4 to 7 to arrive at zero. We note, however, that the rate of decrease in abundance was slightly lower between DBE numbers 5 and 8 . The second pattern showed the abundance of total $O_{\mathrm{o}}$ species to generally decrease as DBE values increased (i.e., Samples 3282, 3247, and 3251). We note a slightly slower rate of decrease (or even a small increase) for total $O_{\mathrm{o}}$ species for DBE numbers 4 to 7 . The maximum peaks accounted for $25-35 \%$ of total $O_{0}$ species (Fig. S4).

Most samples of the first pattern represent Arrow oil residues. Samples with a marked biological contribution and predominant even-over-odd carbon numbers in the short carbon-chain zone re members of the second pattern. The stability of NAFCs to weathering depends on the NAFC carbon-chain length and DBE values (Misiti et al. 2014, Ray et al. 2014, Yang et al. 2017b). NAFCs with a greater number of naphthenic rings are more photo-sensitive than those with fewer rings(King et al. 2014, Leshuk et al. 2016). This difference in photosensitivity could explain why. In general, the saturated congeners are more abundant than unsaturated congeners. On the other hand, NAFCs having more cyclic rings tend to be 
more environmentally persistent and resistant to biodegradation (Misiti et al. 2014). Moreover, biodegradation of NAFCs is sterically hindered by the presence of alkyl substitutions, which prevents $\beta$ oxidation in aliphatic structures and prevents ring opening in cyclic or aromatic structures(Yue et al. 2016). Polycyclic aromatic components are the most photosensitive group ${ }^{29}$. Their photo-oxidation can form abundant NAFCs with certain unsaturated degrees(Yang et al. 2016). Thus, the observed slight increase or slower rate of decrease in abundance for $O_{0}$ species having DBE values of 4 to 7 can be ascribed to the preferable photolysis of hydrocarbons with polycyclic aromatic rings.

\subsubsection{Identified $O_{0}$ species versus oxygen numbers}

Monocarboxylic acids $\left(\mathrm{O}_{2}\right.$ species $)$ are the most abundant oxygen-containing acidic components in crude oil and diluted bitumen (Colati et al. 2013); however, solar radiation and microbial metabolism can add oxygen to the oil components and form higher-order oxygen classes $\left(O_{4}-O_{7}\right)$ (Ray et al. 2014, Vaughan et al. 2016). At the same time, biodegradation can decrease the relative abundance of $O_{1}, O_{3}$, and $O_{4}$ species (Kim et al. 2005, Yue et al. 2016). The $O_{0}$ species discussed above may therefore include a range of oxygen numbers. We distinguished $O_{0}$ species having different oxygen numbers (from 1 to 8 ) to better evaluate $O_{0}$ species variation between sampling sites (Fig. 6). In all samples, $O_{2}$ species represented the most abundant class, followed by, with the exception of the Port Hawkesbury samples, comparable abundances of $O_{3}$ and $O_{4}$ species, then by a pattern of decreased abundance as oxygen number increased. $O_{6}$ to $O_{8}$ species were not detected in most samples. The abundance of $O_{1}$ species was usually less than that of $\mathrm{O}_{4}$ species. The abundance pattern of $\mathrm{O}_{2}$ species between samples was similar to the pattern for total $O_{\mathrm{o}}$ species.

Sample 3247 had the highest abundance of $\mathrm{O}_{2}$, followed by the two samples from Cape Auguet, Sample 3179 from Black Duck Cove, one sample from Indian Cove, and the three samples from Black Duck Cove. Both the source oil, Sample 3281, and the naturally weathered oil, Sample 3282, presented the lowest $\mathrm{O}_{2}$ abundance. The relative percentage of $\mathrm{O}_{2}$ species in all samples varied from $50-70 \%$ when normalised to total $O_{0}$ species (Fig. S5). $O_{0}$ species with higher oxygen numbers usually have a greater water solubility and bioavailability than those with lower oxygen numbers (Islam et al. 2013, Ray et al. 2014). Changes in the relative abundance of $\mathrm{O}_{\mathrm{o}}$ species can occur due to the metabolism of $\mathrm{O}_{3}$ - to $\mathrm{O}_{8}$-NAFCs, oxidation of hydrocarbons, and $O_{1}$ - to $O_{2}$-NAFCs in the natural environment(Yue et al. 2016). Physical abrasion from waves can deplete most $O_{0}$ species having a high water solubility from the stranded oil residues, especially at lower tidal level sites or shores exposed to high wave energy.

\section{Conclusions}

In this study, polar NAFCs were analysed and quantified by HPLC-HRMS. These analyses served to evaluate the natural weathering processes of oil spilled from the Arrow tanker and deposited along the shoreline of Chedabucto Bay, Nova Scotia. In all samples, $O_{0}$ species with a high degree of saturation $(\mathrm{DBE}=0-4)$ were most abundant. All other species had a relatively low abundance. $\mathrm{O}_{2}$ species accounted 
for $50-70 \%$ of total $O_{\mathrm{o}}$ species. Relative to the oil stored in the sunken tanker and the two sediment samples collected near the marina Port Hawkesbury area, the NAFCs within most Arrow oil residuesoriginating from the 1970 spill-were predominantly those of heavier molecular weight. The two samples collected from the Port Hawkesbury reflected the predominance of a biological contribution with a smaller petroleum input as well, reflecting recent petroleum contamination. All Arrow oil residues contained NAFCs having the characteristics of petroleum. NAFCs in the oil residues have experienced severe weathering; therefore, the most resistant components to environmental weathering were remained behind in the residues. The varied abundance of NAFCs in the Arrow oil residues between sites confirmed the importance of site-scale environmental conditions for understanding the natural attenuation of oil spilled in the environment.

\section{Declarations}

\section{Acknowledgments}

This work was funded and supported by the Government of Canada's World Class Tanker Safety System (WCTSS) and Ocean Protection Plan programs. Thanks for Canadian Coastal Guard and all ESTS colleagues providing support for sampling.

\section{Declarations}

Conflicts of interest There are no conflicts to declare.

Funding This work was funded and supported by the Government of Canada's World Class Tanker Safety System (WCTSS) and Ocean Protection Plan programs.

Availability of data and material The authors confirm that the data supporting the findings of this study are available within the article and its supplementary materials.

\section{References}

Cheung SG, Wai HY, Shin PKS, Fahlman B (2010) Fatty acid profiles of benthic environment associated with artificial reefs in subtropical Hong Kong. Mar Pollut Bull 60: 301-313

Clemente JS, Fedorak PM (2005) A review of the occurrence, analyses, toxicity and biodegradation of naphthenic acids. Chemosphere 60: 585-600

Colati KAP, Dalmaschio GP, de Castro EVR, Gomes AO, Vaz BG, RomÃ£o W (2013) Monitoring the liquid/liquid extraction of naphthenic acids in brazilian crude oil using electrospray ionization FT-ICR mass spectrometry (ESI FT-ICR MS). Fuel 108: 647-655

Dunn RJK, Welsh DT, Teasdale PR, Lee SY, Lemckert CJ, Meiziane T (2008) Investigating the distribution and sources of organic matter in surface sediment of Coombabah Lake (Australia) using elemental 
isotopic and fatty acid biomarkers. Cont Shelf Res 28: 2535-2549

Frank RA, Kavanagh R, Bumison BK, Aresnault G, Headley JV, Peru KM, Van Der Kraak G, Solomon KR (2008) Toxicity assessment of collected fractions from an extracted naphthenic acid mixture. Chemosphere 72: 1309-1314

Frank RA, Fischer K, Kavanagh R, Solomon KR (2009) Effect of carboxylic acid content on the acute toxicity of oil sands naphthenic acids. Environ Sci Technol 43: 266-271

Headley JV, Peru KM, Barrow MP (2016) Advances in mass spectrometric characterization of naphthenic acids fraction compounds in oil sands environmental samples and crude oil-A review. Mass Spectrom Rev 35: 311-328

Islam A, Cho Y, Yim UH, Shim WJ, Kim YH, Kim S (2013) The comparison of naturally weathered oil and artificially photo-degraded oil at the molecular level by a combination of SARA fractionation and FT-ICR MS. J Hazard Mater 263, Part 2: 404-411

Katarina Huba A, Gardinali PR (2016) Characterizatoin of a crude oil weathering series by ultrahighresolution mass spectrometry using multiple ionization modes. Sci Total Environ 563-564: 600-610

Kim S, Stanford LA, Rodgers RP, Marshall AG, Walters C, C, Qian K, Wenger LM, Mankiewicz P (2005) Micorbialalteration of the acidic and neutral polar NSO compounds revealed by Fourier transform ion cyclotron resonance mass spectrometry. Org Geochem 36: 1117-1134

King S, Leaf P, Olson P, Ray M, Tarr M (2014) Photolytic and photocatalytic degrada-tion of surface oil from the Deepwater Horizon spill. Chemosphere 95: 415-422

Lee K, Prince RC, Greer CW, Doe KG, Wilson JEH, Cobanli SE, Wohlgeschaffen GD, Alroumi D, King T, Tremblay GH (2003) Composition and toxicity of residual Bunker C fuel oil in intertidal sediments after 30 years. Spill Sci Technol Bull 8: 187-199

Lemkau KL, McKenna A, Podgorski DC, Rodgers RP, Reddy CM (2014) Molecular evidence of heavy-oil weathering following the M/V Cosco Busan spill: Insights from Fourier Transform lon Cyclotron Resonance Mass Spectrometry. Environ Sci Technol 48: 3760-3767

Leshuk T, Wong T, Linley S, Peru KM, Headley JV, Gu F (2016) Solar photocatalytic degradation of naphthenic acids in oil sands process-affected water. Chemosphere 144: 1854-1861

McLafferty FW, Turecek F (1993) Interpretation of Mass Spectra. University Science Books, Mill Vallet, CA Misiti TM, Tezel U, Pavlostathis SG (2014) Effect of alkyl side chain location and cyclicity on the aerobic biotransformation of naphthenic acids. Environ Sci Technol 45: 7431-7437 
Owens EH (1978) Mechanical dispersal of oil stranded in the littoral zone. J Fish Res Borad Can 35: 563572

Owens EH, Humphrey B, Sergy GA (1994) Natural cleaning of oil on two coarse-sediment shorelines on the Arctic and Atlantic coasts of Canada. Spill Sci Technol Bull 1: 37-52

Owens EH, Prince RC, Taylor RB (2008a) Natural attenuation of heavy oil on coarse sediment beaches: Results from Black Duck Cove, Nova Scotia, Canada, over 35 years following the ARROW oil spill, Proceedings of the 31th AMOP Technical Seminar on Environmental Contamination and Response. Environment Canada, Ottawa, ON, Canada, pp. 585-599

Owens EH, Taylor E, Humphrey B (2008b) The persistence and character of stranded oil on coarsesediment beaches. Mar Pollut Bull 56: 14-26

Page JS, Kelly RT, Tang K, Smith RD (2007) Ionization and transmission efficiency in an electrospray ionization-mass spectrometry interface. J Am Soc Mass Spectrom 18: 1582-1590

Ray PZ, Chen H, Podgorski DC, McKenna AM, Tarr MA (2014) Sunlight creates oxygenated species in water-soluble fractions of Deepwater horizon oil. J Hazard Mater 280: 636-643

Rowland SJ, Scarlett AG, Jones D, West CE, Frank RA, Hewitt LM (2011) Sterodial aromatic 'naphthenic acids' in oil sands process-affected water: structural comparisons with environmental estrogens. Environ Sci Technol 45: 9806-9815

Thomas ML (1977) Long term biological effects of Bunker $C$ oil in the intertidal zone. In: Wolfe DA (Editor), Fate and Effects of Petroleum Hydrocarbons in Marine Organisms and Ecosystems. Pergamon Press, New York, NY, pp. 238-245

Vandermeulen JH, Gordon JH (1976) Reentry of 5-year oil stranded Bunker C fuel oil from a low-energy beach into water, sediments, and biota of Chedabucto Bay, Nova Scotia. J Fish Res Borad Can 33: 20022010

Vandermeulen JH, Buckley DE 1985 The Kurdistan oil spill of March 16-17, 1979: Activities and observations of the Bedford Institute of Oceanography Response Team, Bedford Institute of Oceanography, Dartmouth, Nova Scotia

Vandermeulen JH, Singh JG (1994) ARROW oil spill, 1970-90: Persistence of 20yr weathered Bunker C fuel oil. Can J Fish Aquat Sci 51: 845-855

Vanderveen R, Mclnnis R, Brunswick P, Shang D, Headley JV, Peru KM, Hewitt LM (2017) Toxicity of naphthenic acids to invertebrates: Extracts from oil sands process-affected water versus commercial mixtures. Environ Pollut 227: 271-279 
Vaughan PP, Wilson T, Kamerman R, Hagy ME, McKenna A, Chen H, Jeffrey WH (2016) Photochemical changes in water accommodated fractions of MC252 and surrogate oil created during solar exposure as determined by FT-ICR MS. Mar Pollut Bull 104: 262-268

Wang Z, Fingas M, Sergy G (1994) Study of 22-year-old ARROW oil samples using biomarker compounds by GC/MS. Environ Sci Technol 28: 1733-1746

Yang Z, Hollebone BP, Brown CE, Yang C, Wang Z, Zhang G, Lambert P, Landriault M, Shah K (2016) The photolytic behavior of diluted bitumen in simulated seawater by exposed to the natural sunlight. Fuel 186: $128-139$

Yang Z, Hollebone HP, Laforest S, Lambert P, Brown CE, Yang C, Shah K, Landriault M, Goldthorp M (2017a) Occurrence, source and ecological assessment of baseline hydrocarbons in the intertidal marine sediments along the shoreline of Douglas Channel to Hecate Strait in British Columbia. Mar Pollut Bull 122: $450-455$

Yang Z, Zhang G, Hollebone BP, Brown CE, Yang C, Lambert P, Wang Z, Landriault M, Shah K (2017b) Fate of oxygenated intermediates in solar irradiated diluted bitumen mixed with saltwater. Environmental Pollution 231: 622-634

Yang Z, Shah K, Laforest S, Hollebone BP, Lambert P, Brown CE, Yang C, Goldthorp M (2018) A study of the 46-year-old Arrow oil spill: Persistence of oil residues and variability in oil contamination along Chedabucto Bay, Nova Scotia, Canada. J Clean Prod 198: 1459-1473

Yue S, Ramsay BA, Wang J, Ramsay JA (2016) Biodegradation and detoxification of naphthenic acids in oil sands process affected waters. Sci Total Environ 572: 273-279

Zhang G, Yang C, Liu Y, Yang Z, Wang Z, Landriault M, Hollebone BP, Lambert P, Brown CE (2014) Improved approach to quantitatively and qualitatively characterize carboxylic acid isomers in commercial naphthenic acids mixture by HPLC-high resolution Orbitrap mass spectrometer technique, Proceedings of the $37^{\text {th }}$ AMOP Technical Seminar on Environmental Contamination and Response. Environment and Climate Change Canada, Ottawa, ON, pp. 848-869

\section{Tables}

Table 1 Sample information including sampling date, site characteristics, and location. 


\begin{tabular}{lll}
\hline ESTS code & Sample information & Longitude (N), Latitude (W) \\
\hline $\mathbf{2 0 1 6 / 0 6 / 0 8 - 3 1 6 3}$ & Cape Auguet, oil under rocks & $45.46867 \mathrm{~N}, 61.02216 \mathrm{~W}$ \\
\cline { 1 - 1 } $\mathbf{2 0 1 6 / 0 6 / 0 8 - 3 1 6 5}$ & Cape Auguet, oil under rocks & $45.4686 \mathrm{~N}, 61.02224 \mathrm{~W}$ \\
\hline $\mathbf{2 0 1 6 / 0 6 / 0 8 - 3 1 7 9}$ & Black Duck Cove, rocks, UIZ & $45.27284 \mathrm{~N}, 61.03024 \mathrm{~W}$ \\
\hline $\mathbf{2 0 1 6 / 0 6 / 0 8 - 3 1 8 4}$ & Black Duck Cove, rocks, UIZ & $45.27266 \mathrm{~N}, 61.03056 \mathrm{~W}$ \\
\hline $\mathbf{2 0 1 6 / 0 6 / 0 8 - 3 1 8 6}$ & Black Duck Cove, oil under rocks, UIZ & $45.27257 \mathrm{~N}, 61.03076 \mathrm{~W}$ \\
\hline $\mathbf{2 0 1 6 / 0 6 / 0 8 - 3 1 9 3}$ & Black Duck Cove, oil on rocks, UIZ & $45.26755 \mathrm{~N}, 61.03476 \mathrm{~W}$ \\
\hline $\mathbf{2 0 1 6 / 0 6 / 0 8 - 3 2 1 6}$ & Indian Cove, oil on rocks, UIZ & $45.34243 \mathrm{~N}, 61.10448 \mathrm{~W}$ \\
\hline $\mathbf{2 0 1 6 / 0 6 / 0 8 - 3 2 4 7}$ & Port Hawkesbury near marina, sediment, LIZ & $45.61484 \mathrm{~N}, 61.36554 \mathrm{~W}$ \\
\hline $\mathbf{2 0 1 6 / 0 6 / 0 8 - 3 2 5 1}$ & Port Hawkesbury at marina, sediment, MIZ & $45.61487 \mathrm{~N}, 61.36548 \mathrm{~W}$ \\
\hline $\mathbf{2 0 1 6 / 0 6 / 2 2 - 3 2 8 1}$ & Source oil samples from the sunken tanker in 2015 & $/$ \\
\hline $\mathbf{2 0 1 6 / 0 6 / 2 2 - 3 2 8 2}$ & Naturally weathered source oil spilled in 2015 & $/$ \\
\hline
\end{tabular}

Note: LIZ: lower intertidal zone; UIZ: upper intertidal zone; and MIZ: middle intertidal zone. "/": data are not available.

Figures 


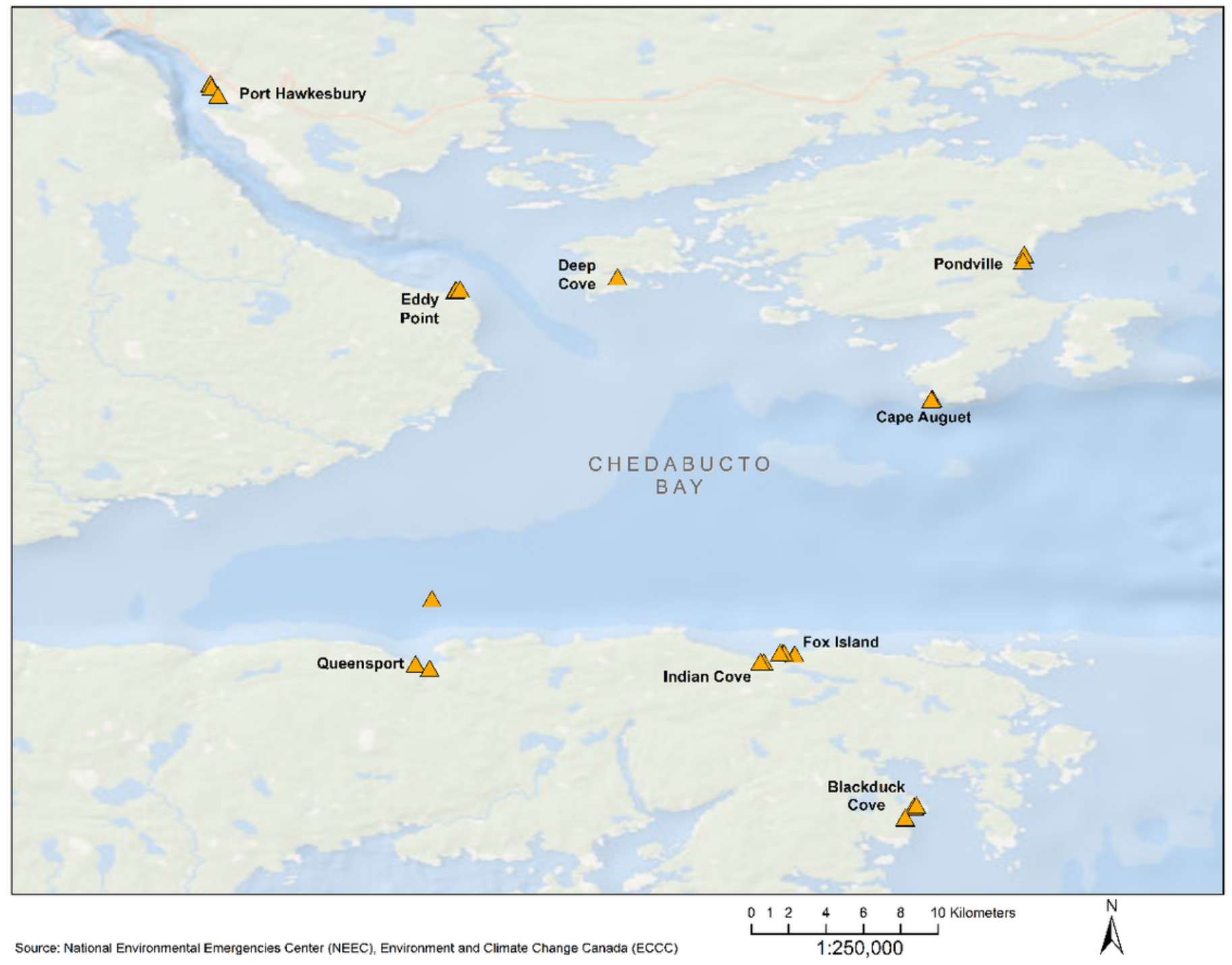

Figure 1

Sampling locations around Chedabucto Bay, Nova Scotia, Canada. 
(a)
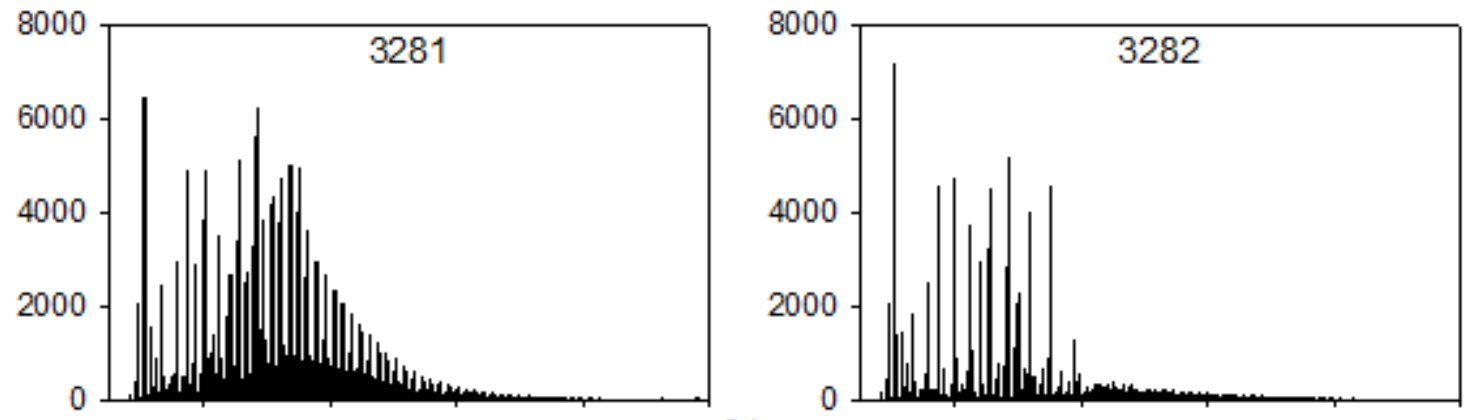

(b)
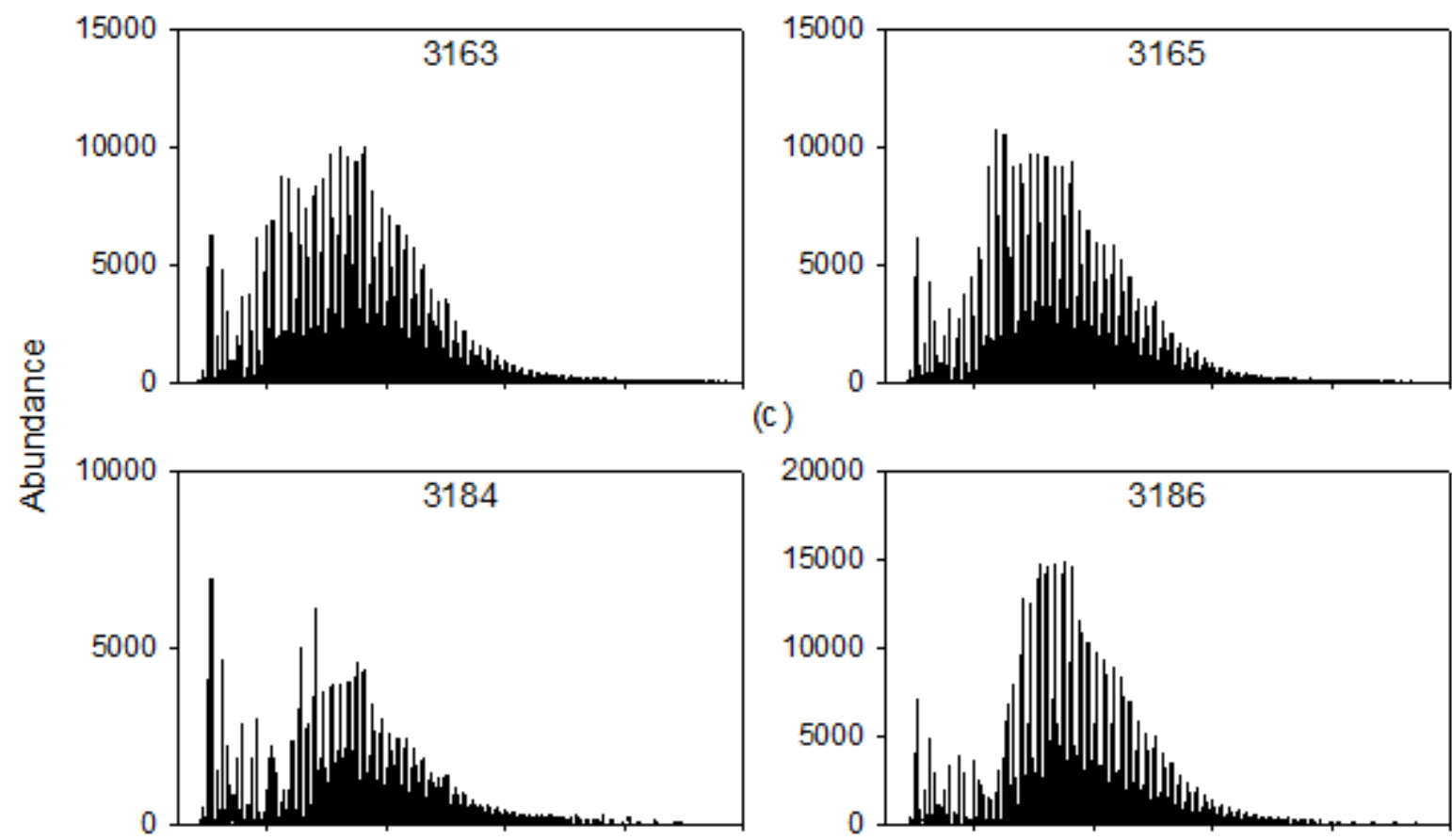

(c)

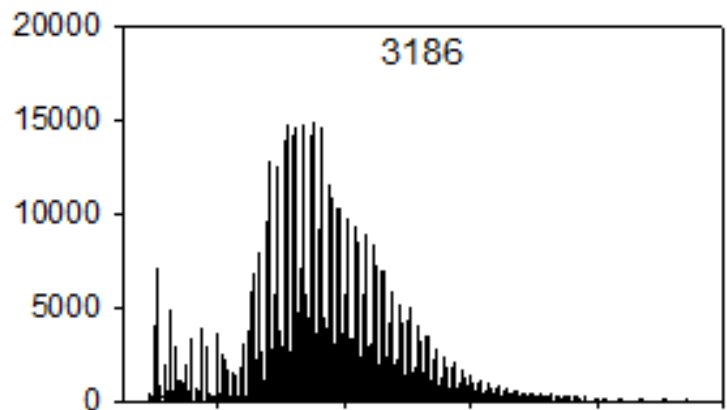

(d)
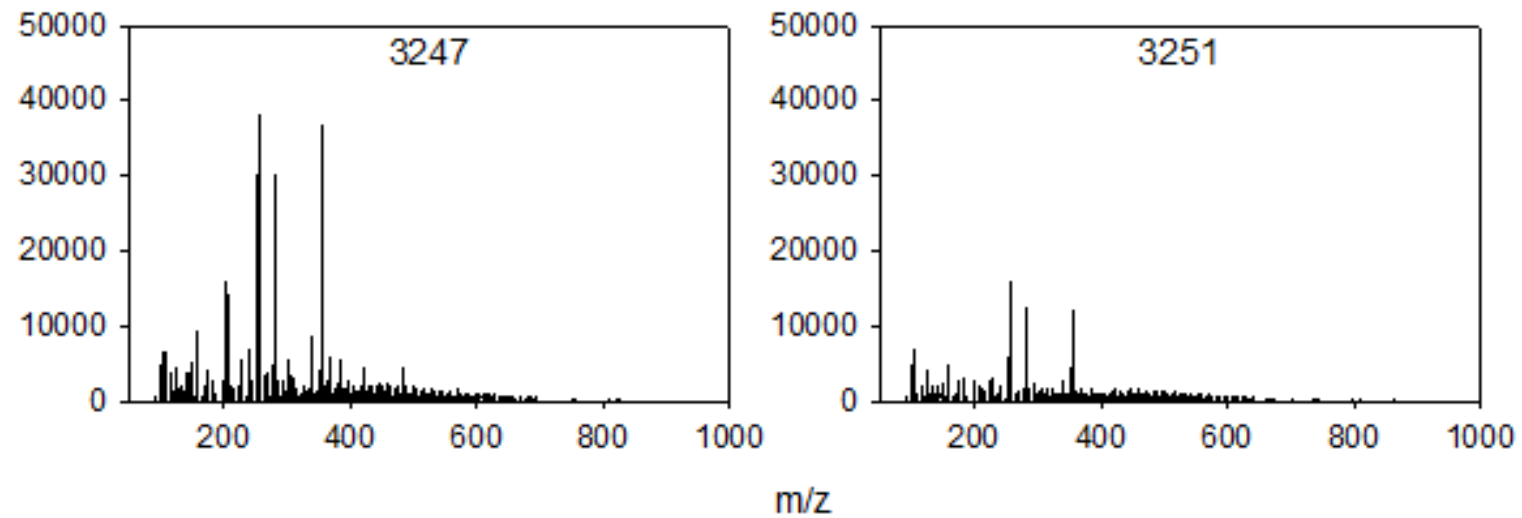

Figure 2

Average negative-ion ESI Orbitrap mass spectra of the acidic extractable fractions for representative samples. (a) Source oil from the Arrow spill; (b) Arrow oil residues collected from Cape Auguet; (c) Arrow oil residues collected from Black Duck Cove; (d) sediments from the Port Hawkesbury marina area. 

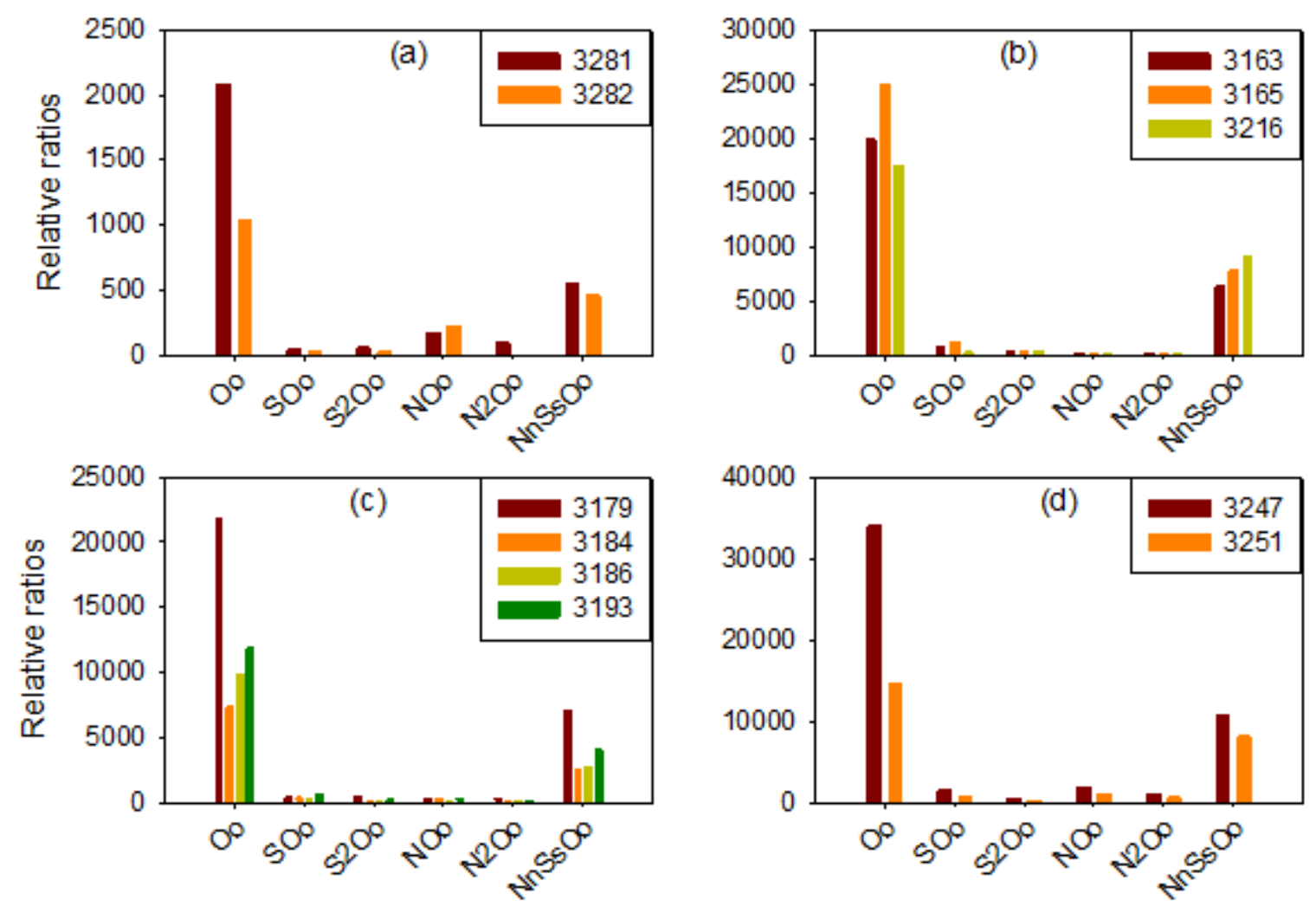

Figure 3

Relative ratios of the different species subtotals. (a) Source oil from the Arrow spill; (b) Arrow oil residues collected from Cape Auguet; (c) Arrow oil residues collected from Black Duck Cove; (d) sediments from the Port Hawkesbury marina area. 
(a)

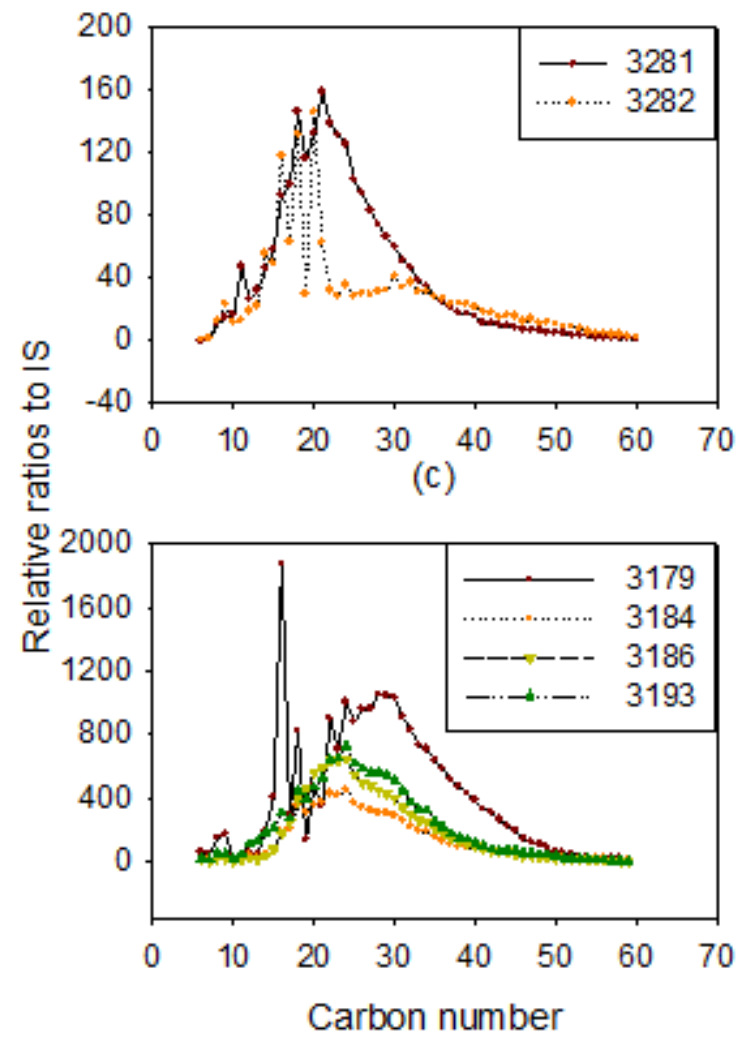

(b)
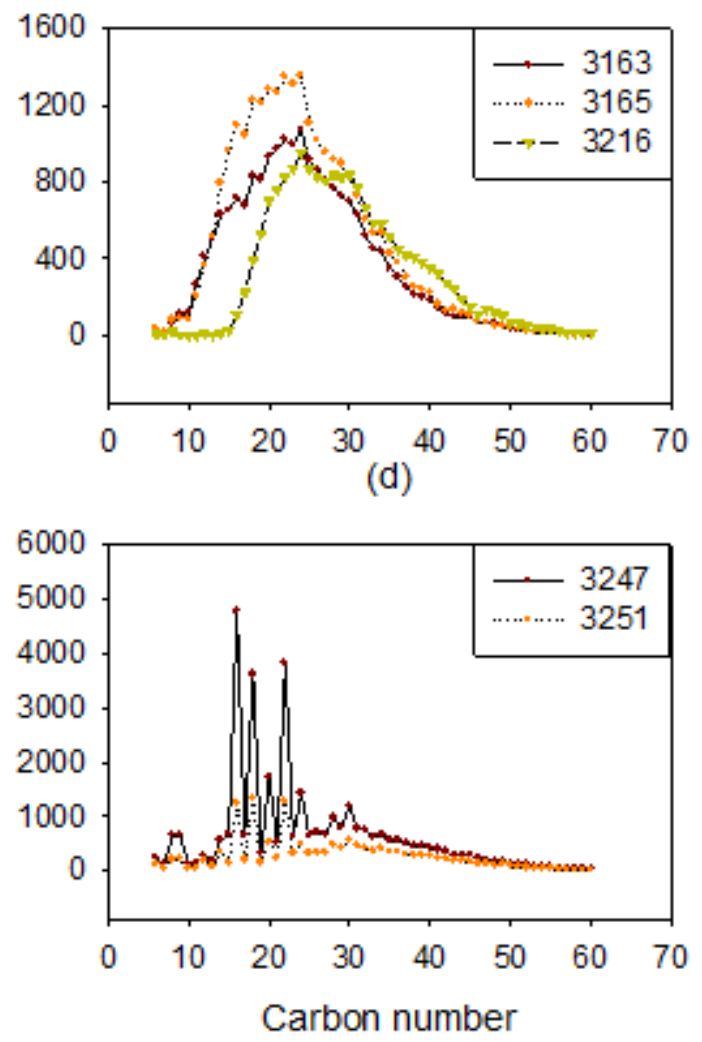

\section{Figure 4}

Distribution profiles of total Oo species versus carbon number. (a) Source oil from the Arrow spill; (b) Arrow oil residues collected from Cape Auguet; (c) Arrow oil residues collected from Black Duck Cove; (d) sediments from the Port Hawkesbury marina area. 
(a)

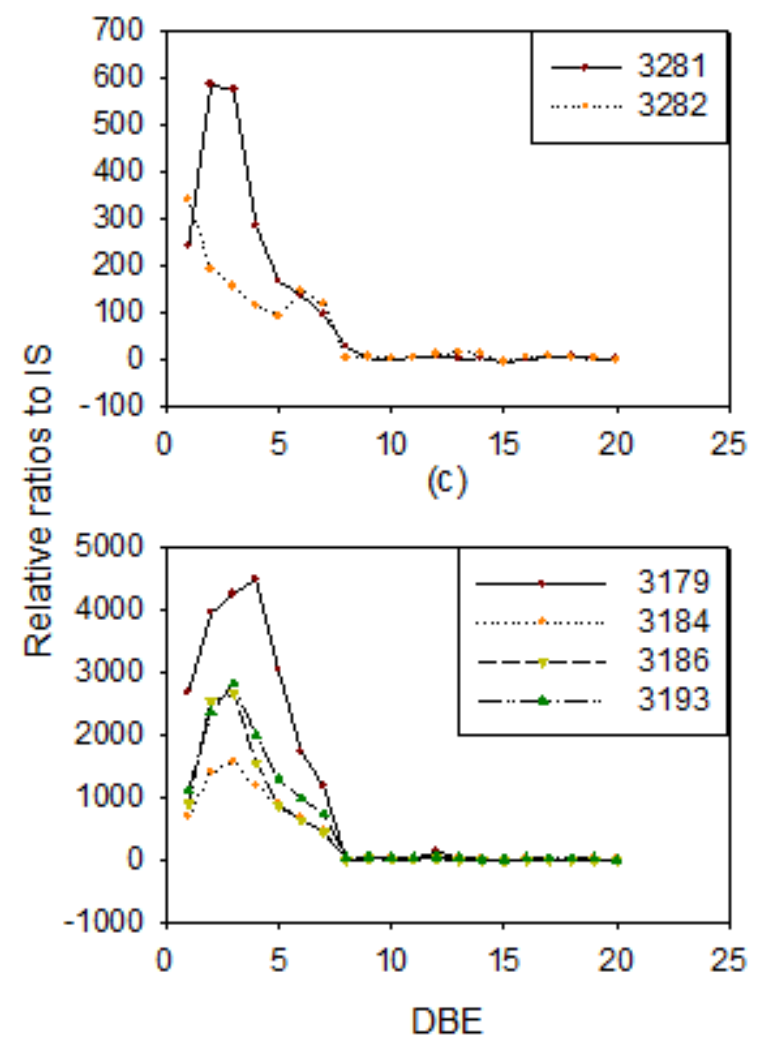

(b)
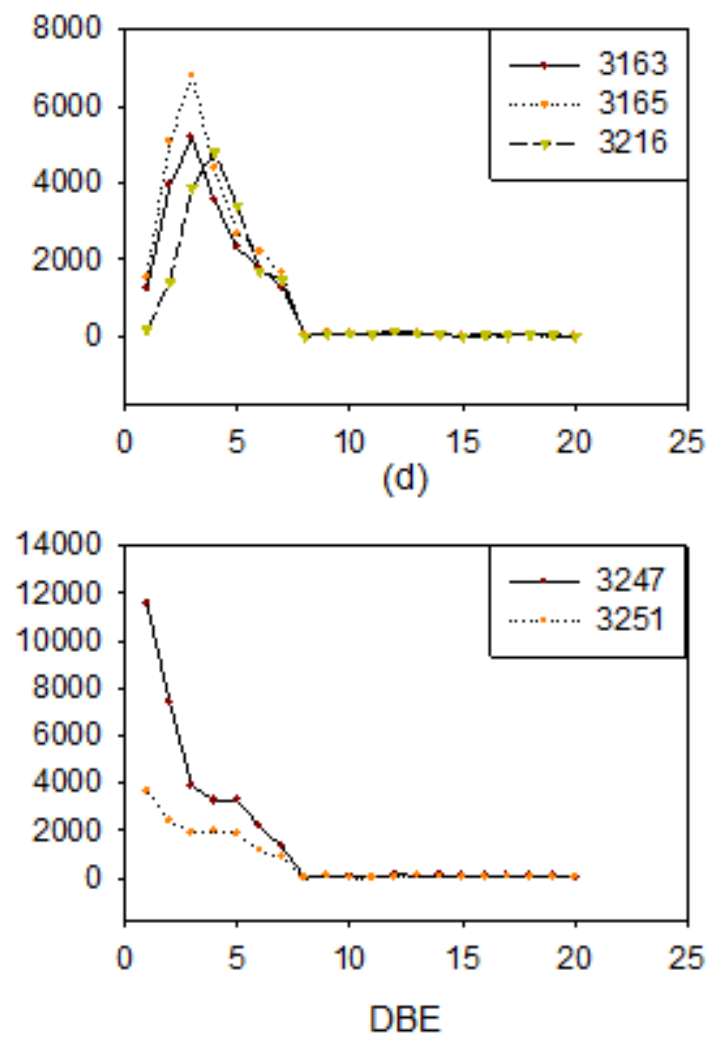

Figure 5

Distribution profiles of total Oo species versus DBE number. (a) Source oil from the Arrow spill; (b) Arrow oil residues collected from Cape Auguet; (c) Arrow oil residues collected from Black Duck Cove; (d) sediments from the Port Hawkesbury marina area. 

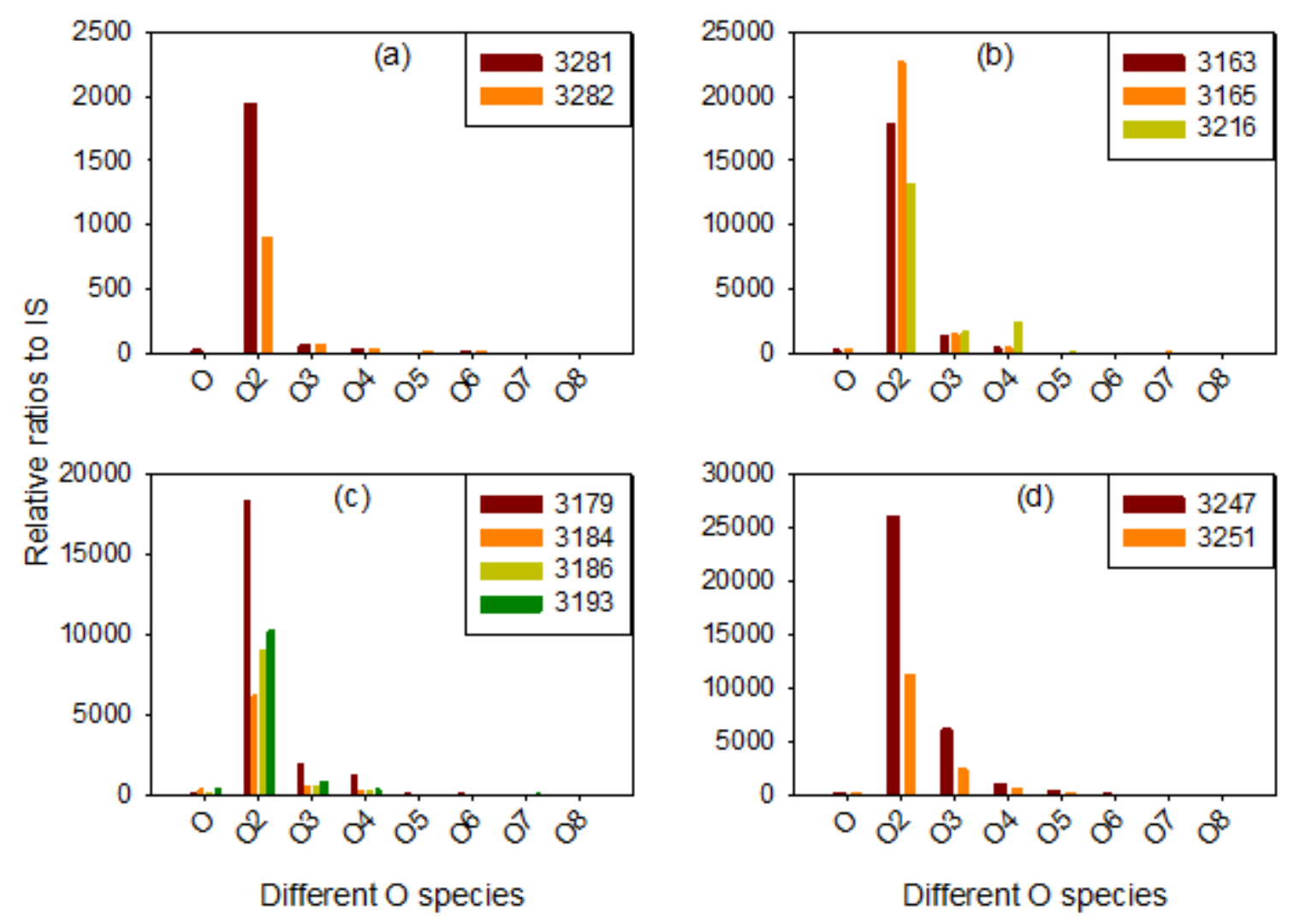

\section{Figure 6}

Relative ratios of subtotal Oo species of different oxygen numbers. (a) Source oil from the Arrow spill; (b) Arrow oil residues collected from Cape Auguet; (c) Arrow oil residues collected from Black Duck Cove; (d) sediments from the Port Hawkesbury marina area.

\section{Supplementary Files}

This is a list of supplementary files associated with this preprint. Click to download.

- Supportinginformation2020.docx 